\title{
Fertility in men diagnosed with ankylosing spondylitis - a population-based study
}

\author{
Mihaela Cosmina Micu' ${ }^{1}$, Romeo Micu², Roxana Ionescu ${ }^{3}$ \\ ${ }^{1}$ Rheumatology Division, $2^{\text {nd }}$ Rehabilitation Department, \\ Rehabilitation Clinical Hospital, Cluj-Napoca, Romania \\ ${ }^{2}$ Department of Human Assisted Reproduction, $1^{\text {st }}$ Gynecology Clinic, \\ "Iuliu Hatieganu" University of Medicine and Pharmacy, Cluj-Napoca, Romania \\ ${ }^{3}$ Department of Internal Medicine and Rheumatology, Sf. Maria Hospital, \\ "Carol Davila" University of Medicine and Pharmacy, Bucharest, Romania
}

\begin{abstract}
Introduction. Disease activity, drug exposure, infectious factors, smoking, excessive alcohol intake and stress were identified to have a negative impact on spermatogenesis and gonadal function. Population-based studies on fertility in ankylosing spondylitis (AS) men are lacking. It is not known, if men with AS have a reduced number of children. Our objective was to determine fertility in a masculine AS group by determining the family size.

Patients and methods. Men diagnosed with AS responded to a questionnaire comprising of several domains, focused on the preconception/conception period. Demographic parameters, disease related information, sexual relationship status, infertility treatment use in the couple, time to pregnancy achievement (TTP), comorbidities, exposure to gonadotoxic medication or radiation in the past, smoking, number of pregnancies and children born in the couple and couples' decision to limit family size were registered.

Results. Out of 122 AS patients, 76 accepted to participate to the interview. Hundred-eleven pregnancies were registered in the couples and 99 children were born. Forty-two men (55.26\%) with AS onset fathered 58 children and $34(44.73 \%)$ men fathered 41 children before AS onset. No statistic difference was found between the 2 subgroups when average number of children/ family was compared (1.38 vs 1.20$)$. A statistic significant number of men, with AS onset at conception (14/42; 33.33\%), decided to limit the family size $(p=0.02)$.

Twenty-seven patients $(35.52 \%)$ recalled a decrease in sexual desire. In $19(25 \%)$ of them, this phenomenon was linked to the disease burden. Decrease of sexual desire in the 2 groups showed a borderline statistic significant difference, $p=0.051$.

No statistic significant difference was obtained when TTP in the two groups were compared.

Conclusion. A lower fertility in AS patients was described in our group in comparison to general population and this phenomenon could be linked mostly to psychological factors impacting family size decision. No correlation between the low fertility and disease presence at conception was found.
\end{abstract}

Keywords: men, fertility, average number of children, ankylosing spondylitis

\section{INTRODUCTION}

Ankylosing spondylitis (AS) is a chronic inflammatory disease affecting the axial skeleton, entheses and occasionally the peripheral joints and extra-articular organs. It has a higher prevalence in men in comparison to women $(1,2)$. As it develops mainly during peak reproductive years, quality of life, including sexual function and fertility status, may be negatively influenced $(2,3)$.

Impairment of reproduction function in patients with chronic rheumatic inflammatory diseases is mainly linked to factors depending on the AS pro- cess itself, drug management and on the patients' ability to cope with the disease (4).

Male fertility refers to a male ability to cause pregnancy in a fertile female and is related mainly to the number of born children (5). Male fecundity (his biological capacity to reproduce) is a component of the fertility rate.

There is no way of measuring the male fertility component directly, but several markers have been used in clinical practice and studies. Among biological indicators, sperm quality and quantity isolate the male component of fertility. Sperm analysis has 
proved to be superior to other markers, like serum levels of the gonadotropins follicle stimulating hormone (FSH), luteinizing hormone (LH) and inhibin $\mathrm{B}$, in predicting a conception (6-9).

Indeed, studies using time-to-pregnancy (TTP) measurements may identify couple sub-fertility when pregnancy achievement takes longer than 12 months, and TTP has been shown to correlate with sperm quality and quantity as well as with sexual activity (10-12).

Fertility studies in men diagnosed with different rheumatic diseases have focused on the evaluation of sperm parameters, gonadal hormonal function, testicular alterations and anti-sperm antibodies. Disease activity, drug exposure, infectious factors, smoking, excessive alcohol intake and stress were identified to have a negative impact on spermatogenesis and gonadal function $(4,13,14)$.

In theory, a normal spermiogram represents a surrogate measure for normal fertility which needs further confirmation due to pregnancy achievement in the couple (15). Infertility affects up to $12 \%$ of all men, but abnormal semen production is only responsible for approximately $50 \%$ of male factor infertility (16-18). In the absence of sperm analysis it is more difficult to estimate men fertility. An indirect parameter would be the calculation of the average number of children in the family.

By now, no population-based study on fertility in AS men was performed in Romania and no population-based study has ever investigated, if men with rheumatic diseases have a reduced number of children.

Our objective was to determine fertility in a masculine AS group by determining the family size.

TABLE 1. Presents the demographic data of the group.

\begin{tabular}{|l|c|}
\hline Group parameters & \\
\hline Nr. patients & 76 \\
\hline AS axial involvement $-\mathrm{Nr}[\%]$ & $53[69.73]$ \\
\hline AS axial and peripheral $-\mathrm{Nr}[\%]$ & $23[30.26]$ \\
\hline mean age $(\mathrm{y} \pm \mathrm{SD})$ & $30 \pm 5.017$ \\
\hline marital status $-\mathrm{Nr}[\%]$ & \\
\hline maried & $72[94.73]$ \\
\hline not maried & $4[5.26]$ \\
\hline AS dg at conception & $58[58.58]$ \\
\hline disease duration $(\mathrm{y} \pm \mathrm{SD})$ & $4 \pm 2.83$ \\
\hline Smokers $-\mathrm{Nr}[\%]$ & $23[23.23]$ \\
\hline therapy & \\
\hline $\mathrm{NSAIDs}-\mathrm{Nr}[\%]$ & $58[58]$ \\
\hline SSZ - Nr[\%] & $5[5.26]$ \\
\hline
\end{tabular}

Legend: SD - standard deviation, AS - ankylosing spondylitis, $\mathrm{Nr}$ - number, NSAIDs - nonsteroidal anti-inflammatory drugs, SSZ - sulphasalazine.
TABLE 2. Presents the reproduction data in the groups

\begin{tabular}{|l|c|c|c|}
\hline Reproduction data & all over & $\begin{array}{c}\text { fathers' } \\
\text { with AS }\end{array}$ & $\begin{array}{c}\text { fathers' } \\
\text { without AS }\end{array}$ \\
\hline Pregnancies Nr[\%] & $111[100]$ & $66[59.45]$ & $45[40.54]$ \\
\hline Children $\mathrm{Nr}[\%]$ & $99[89.18]$ & $58[58.58]$ & $41[41.41]$ \\
\hline Nr. children/father & 1.32. & 1.38. & 1.2. \\
\hline Elective abortion $\mathrm{Nr}[\%]$ & $7[6.3]$ & $4[3.6]$ & $3[2.70]$ \\
\hline $\begin{array}{l}\text { Spontaneous abortion } \\
\text { Nr[\%] }\end{array}$ & $4[3.6]$ & $3[2.70]$ & $1[0.9]$ \\
\hline $\begin{array}{l}\text { Therapeutic abortion } \\
\mathrm{Nr}[\%]\end{array}$ & $1[0.9]$ & $1[0.90]$ & $0[0.0]$ \\
\hline TTP >12 months & $6[6.06]$ & $3[5.17]$ & $3[7.31]$ \\
\hline $\begin{array}{l}\text { Fertility treatments } \\
\mathrm{Nr}[\%]\end{array}$ & $1[0.9]$ & $1[0.9]$ & $0[0.0]$ \\
\hline $\begin{array}{l}\text { Erectile dysfunctions } \\
\mathrm{Nr}[\%]\end{array}$ & $0[0]$ & $0[0.0]$ & $0[0.0]$ \\
\hline $\begin{array}{l}\text { Sexual desire decrease } \\
\mathrm{Nr}[\%]\end{array}$ & $27[35.52]$ & $19[32.75]$ & $8[34.78]$ \\
\hline
\end{tabular}

Legend: $\mathrm{Nr}$ - number, AS - ankylosing spondylitis,

TTP - time to pregnancy achievement.

\section{PATIENTS AND METHODS}

Men diagnosed with AS according to New York diagnostic criteria were selected to respond to a questionnaire comprising of several domains, focused on the preconception/conception period. Following information was recorded: demographic parameters (subjects age, marital status, diagnose of the disease in relation to the conception period), disease related information (disease onset- years, subtype of AS - axial or axial and peripheral involvement, disease activity Bath Ankylosing Spondylitis Disease Activity Index BASDAI, any AS specific therapy intake - including preconception period), sexual relationship status (presence of erectile dysfunction, sexual desire decrease), infertility treatment use in the couple and TTP, comorbidities (varicocele, urlian virus infection in adulthood), smoker status, any exposure to gonadotoxic medication or radiation in the past, number of pregnancies and children in the couple and couples' decision to limit family size.

The questionnaire was taken home and data was completed by the couple. Fertility was calculated as being reflected by the number of children/patients' family.

The study was approved by the ethics committee (Rehabilitation Clinical Hospital Cluj-Napoca, N 2394/3.04.2017) and informed written consent was obtained from patients.

\section{STATISTICAL ANALYSIS}

The assessment of the normality of data was performed using Shapiro-Wilk test. Descriptive statis- 
tics was performed for the continuous and categorical variables in the two groups, and results were expressed as mean \pm standard deviation (SD) or number of cases and percent. Chi-square test with a significance of 0.05 was used to compare groups. The statistical analysis was performed using SPSS software version 22 and Microsoft Office Excel 2010.

\section{RESULTS}

Out of 122 AS patients, 76 accepted to participate to the interview (53 diagnosed with axial disease and 23 with axial and peripheral disease). Hundred-eleven pregnancies were registered in the couples and 99 children were born. Forty-two men $(55.26 \%)$ with AS onset fathered 58 children and $34(44.73 \%)$ men fathered 41 children before AS onset. Five ( $6.57 \%$ ) men fathered children both before and after disease onset. Twenty-three men fathered more than 1 child (30.66\%). Fifty-one men fathered 1 child, 16 men fathered 2 children, 4 men fathered 3 children and one fathered 4 children; 4 men did not father children.

Twelve men with AS onset have fathered more than 1 child while 11 men without AS onset fathered more than 1 child ( $52.17 \%$ vs $47.82 \%$ ).

No statistic significant difference was found in the mean age between men that conceived during the disease course or before ( $30 \pm 4.64$ vs $30 \pm 5.49$ years). No statistic difference was found between the 2 subgroups when average number of children/family was compared (1.38 for men with AS onset vs 1.20 for men without AS onset). Fourteen/42 (33.33\%) men with AS onset at conception were counseled (eg. medical staff, family, friends) not to have children or to limit the family size, as AS would be inherited by the next generation. Statistic significant difference of $p=0.02$ was found between the 2 groups when decision to limit family size analysis was made.

Exposure to medication was present only in men conceiving after disease onset. In this sub-group, NSAIDs prescription was recorded, but schedule of administration, type and dosage could not be accurately determined. Five patients recalled continuous SSZ treatment at the time of conception; they fathered 5 children and one couple recalled TTP $>12$ months.

Overall, 27 patients $(35.52 \%)$ recalled a decrease in sexual desire. The phenomenon was identified as being intermittent and recognized as being "from time to time" or "sometimes". In 19 (25\%) patients, this phenomenon was linked to the disease burden. Decrease of sexual desire in the 2 groups showed a borderline statistic significant difference, $p=0.051$.

Fourteen smokers were identified among the fathers' with AS onset, representing $60.86 \%$ of the total smokers. No erectile or other organic sexual relationship dysfunction was identified. BASDAI was available in 5 patients showing active disease in 3 of them. TTP $>12$ months was $5.17 \%$ in the couples where the father was already diagnosed with AS vs $7.31 \%$ in the couples with healthy males. No statistic significant difference was obtained when TTP in the two groups were compared. One woman received fertility treatment. Her partner had normal sperm parameters.

\section{DISCUSSION}

We performed a retrospective interview-based analysis on fertility in AS men. To our knowledge, this is the first population-based study in Romania. Regardless of the fact that conception took place before or after disease onset, our study showed an overall lower average number of children/family (1.32) in comparison to general population.

Official European organizations report a decrease in the average number of children in married couples from 2.33 in 1960 to 2.01 in 1977 , reaching a level of 1.9 in 2017 (19). In the United States, the same parameter dropped from 2.3 in 1970 to 1.9 in 2017 (20). In Romania, the National Institute of Statistics (INS) reports identify an average number of children in families of 2.19 in 1989 with a decrease to 1.4 in 2013 (21).

Studies reviewing men infertility meta-analysis and population-based analyses found that geographic distribution of couples infertility due to male factor ranged from $20 \%$ to $70 \%$ while the percentage of infertile men ranged from $2-5 \%$ to $12 \%$. The highest rates were identified in in Africa and Central/Eastern Europe (18).

Surprisingly, in our study, men conceiving before disease onset had a lower fertility (1.20) in comparison to those conceiving after AS onset (1.38). One third of the fathers with AS onset at conception recognized external influence on the couple decision to have children and this parameter showed to be statistically significant. Indeed, 27 (35.52\%) patients in the entire group recalled a decrease in sexual desire from time to time. The majority (19/27) of patients 
linked this phenomenon to the disease burden. A borderline statistic significance was obtained for this parameter analysis, but the number of included patients was low. At this point, one could hypothesize that sexual desire decrease and family size restriction counseling could have been some of the trigger factors for a lower number of children in the family in our groups. In addition, we may speculate that AS onset after the first child could have negatively influenced the couples' decision to have more children (risk of inheritance).

It is known by now, that sexual relationships can be affected in patients with inflammatory rheumatic diseases and this may impact fertility and fecundity. In a study on 612 AS patients ( $72 \%$ men), $38 \%$ of them reported a disease impact, ranging from moderate to extreme, on the sexual relationship. Other identified concurrent factors were pain, poor physical function, higher disease activity, anxiety and depression, unemployment and poor self-efficacy (22). Apart from the lower sex desire, none of the above mentioned factors were mentioned by our group; possibly our study groups were younger and had a more recent disease onset in comparison to other studies.

Indeed, no specific erectile dysfunction was identified through interview, regardless of the group affiliation. In other studies, sexual dysfunction was found to affect about $20-50 \%$ of the general population and is related to older age (23).

Other known concurrent factors impacting fertility would be disease activity, therapy exposure and history of comorbidities like varicocele or urlian virus infection in adulthood $(4,14)$.

In our study, we could not appreciate the impact of active AS on the group fertility. Not all clinical records certifying AS activity evaluation were available for the purpose of this study. Hypothetically, some of the men with AS onset at conception could have had a pre-conception high disease activity. We were able to find BASDAI records in only 5 patients, 3 of them showing an active disease status. Disease activity scoring was done within a range of 2-4 months pre-conception.

In inflammatory conditions, higher TNF alpha concentrations are detected in the seminal fluid and adverse effects on spermatogenesis have been reported but information is controversial (24-27). A study performed in vitro, showed that sperm cell exposure to high concentrations of TNF alpha may induce an important loss of their functional and ge- nomic integrity (25). In contrast to this, another prospective study conducted by Micu et al on 20 active AS patients (high activity documented in the last 6 months) who were selected to receive TNFi therapy, showed similar sperm parameters with the control group, consisting of healthy age matched sperm donors (28). Indeed, chronic inflammation may affect the hypothalamic-pituitary-gonadal axis and subsequently the gonadal function $(29,30)$. Normal gonadal function was found in men with AS, with the exception of patients with varicocele, who showed multiple sperm abnormalities (31-33). In our group, just 1 patient recalled an episode of infectious parotitis in adulthood. Varicolele diagnosis was not confirmed in any of the groups.

Fourty-two men diagnosed with AS before conception recalled NSAIDs exposure but medical records including the type, dosage and administration regimens were missing. Whether NSAIDs can in fact impair fertility in men with rheumatic diseases has not been completely clarified, the number of studies showing no harmful impact on spermatogenesis being small (4). In a prospective longitudinal controlled study, AS patients were exposed to continuous regimen of different NSAIDs, daily administration and highest tolerated dose, for at least 6 months prior to sperm analysis. Twelve patients were exposed to NSAIDs mono-therapy, 7 had additional therapy with Sulfasalazine (SSZ) and 4 a combination of SSZ and Methotrexate (MTX). Sperm analysis showed comparable results in patients and healthy age matched controls (28). The same conclusions are supported by another study on 20 AS patients treated with NSAIDs, where sperm parameters were normal (31). In contrast with this data, an observational study performed on 1376 men attending an infertility clinic, detected a NSAIDs (mainly Aspirin) dose-dependent decrease in seminal volume, sperm concentration, quality and motility (34).

In our group, only 5 patients recalled continuous SSZ treatment at the time of conception. They fathered 5 children and 1 couple recalled TTP $>12$ months. Overall, TTP $>12$ months was present in 6 $(6.06 \%)$ pregnancies, equally divided between the 2 sub-groups. SSZ can produce temporary oligospermia, but this phenomenon was mostly observed in inflammatory bowel disease (IBD) patients. After 2-3 months of medication cessation, spermatogenesis recovers, and in some cases successful conception can be achieved (35-40). In animal studies, the 
sulfapyridine metabolite showed to induce transient infertility in a dose dependent way. Infertility was not influenced by folic acid supplementation (35). However, our previous prospective controlled study on AS patients showed no alteration of sperm parameters during longstanding therapy with SSZ in 11 patients with active AS (3 g/daily for 2 years), (28).

Our study was the first one to evaluate fertility in AS patients showing a lower rate in comparison to general population in this group. Psychological factors and reduced sexual desire showed to play a more important role in family size restriction, in comparison to other factors.
The limits of the study were the retrospective collection of interview- based data and the small number of patients. Delivered information may have been biased by errors in recalling the facts.

In conclusion, a lower fertility in AS patients was described in our group in comparison to general population and this phenomenon could be linked mostly to psychological factors impacting family size decision. No correlation between the low fertility and disease presence at conception was found. Future prospective controlled studies on larger AS patient cohorts are required for a thorough fertility evaluation.

Conflict of interest: none declared Financial support: none declared

\section{REFERENCES}

1. Feldkeller E., Khan M.A., van der Heijde D., van der Linden S., Braun J. Age at disease onset and diagnosis delay in HLAB27 negative vs. positive patients with ankylosing spondylitis. Rheumatol Int 2003;23:61-6.

2. Gran J.T., Husby G., Hordvik M. Prevalence of ankylosing spondylitis in males and females in a young middle aged population of Tromso" , northern Norway. Ann Rheum Dis 1985;44:359-67.

3. Cakar E., Dincer U., Kiralp M.Z. et al. Sexual problems in male ankylosing spondylitis patients: relationship with functionality, disease activity, quality of life, and emotional status. Clin Rheumatol 2007;26:1607_1.

4. Ostensen M. Sexual and reproductive health in rheumatic disease. Nature Reviews Rheumatology 2017; 13, 485-493.

5. http://wikipedia.com

6. Jensen T.K., Andersson A.M., Hjollund N.H., Scheike T., Kolstad H. et al. Inhibin B as a serum marker of spermatogenesis: correlation to differences in sperm concentration and follicle-stimulating hormone levels. A study of 349 Danish men. J Clin Endocrinol Metab 1997; 82: 4059-63.

7. Yalti S., Gurbuz B., Ficicioglu C. Serum levels of inhibin B in men and their relationship with gonadal hormones, testicular volume, testicular biopsy results and sperm parameters. J Obstet Gynaecol 2002; 22: 649-54.

8. Mabeck L.M., Jensen M.S., Toft G., Thulstrup M., Andersson M. et al. Fecundability according to male serum inhibin $B-a$ prospective study among first pregnancy planners. Hum Reprod 2005; 20: 2909-15.

9. Meeker J.D., Godfrey-Bailey L., Hauser R. Relationships between serum hormone levels and semen quality among men from an infertility clinic. J Androl 2007; 28: 397-406.

10. Rachootin P., Olsen J. The risk of infertility and delayed conception associated with exposures in the Danish workplace. J Occup Med 1983; 25: 394-402.

11. Levine R.J. Monitoring fertility to detect toxicity to the male reproductive system. Reprod Toxicol 1988; 2: 223-7.

12. Weinberg C.R., Baird D.D., Wilcox A.J. Sources of bias in studies of time to pregnancy. Stat Med 1994; 13: 671-81.

13. Tiseo B.C., Cocuzza M., Bonfa E., Srougi M. \& Silva C.A. Male fertility potential alteration in rheumatic diseases: a systematic review. Int. Braz. J. Urol 2016; 42:11-21.

14. Micu M.C., Ostensen M., Villiger P.M., Micu R., lonescu R. Paternal exposure to antirheumatic drugs- What physicians should know. Review of the literature. Semin Arthritis Rheum 2018; pii: S0049-0172(17)30747-3.

15. Cooper T.G., Noonan E., Von Eckardstein S., Auger J., Baker H.W., Behre H.M., Haugen T.B., Kruger T., Wang C.

„World Health Organization reference values for human semen characteristics". Human Reproduction Update 2009; 16 (3): 231-45.

16. Singh K., Jaiswal D. Human male infertility: a complex multifactorial phenotype. Reprod Sci 2011;18 (5):418-25.

17. Lotti F., Maggi M. „Ultrasound of the male genital tract in relation to male reproductive health". Human Reproduction Update 2014; 21 (1): 56-83.

18. Agrawal A., Mulgund A., Hamada A. Chyatte M.R. A unique view on male infertility around the globe. Reproductive Biology and Endocrinology 2015; 13:37.

19. http://ec.europa.eu (source verified in June 2018)

20. http://www.census.gov (Source: U.S. Census Bureau, Current Population Survey, Annual Social and Economic Supplements, 1955, 1960, 1965 and 1970 to 2017; source verified in June 2018)

21. http://INS.ro (source verified in June 2018)

22. Healey E.L. et al. Ankylosing spondylitis and its impact on sexual relationships. Rheumatology (Oxford) 2009; 48:1378-1381.

23. Laumann E., Paik A. \& Rosen R.C. Sexual dysfunction in the United States: prevalence and predictors. JAMA 281, 537-544 (1999).

24. Lampiao F., du Plessis S.S. TNF-alpha and IL-6 affect human sperm function by elevating nitric oxide production. Reprod Biomed Online 2008;17:628_31.

25. Said T.M., Agarwal A., Falcone T. et al. Infliximab may reverse the toxic effects induced by tumor necrosis factor alpha in human spermatozoa: an in vitro model. Fertil Steril 2005; 86:1665_73.

26. Estrada L.S., Champion H.C., Wang R. et al. Effect of tumor necrosis factor-alpha (TNF-alpha) and interferon-gamma(IFNgamma) on human sperm motility, viability and motion parameters. Int J Androl 1997;20:237-42.

27. Perdichizzi A., Nicoletti F., La Vignera F. et al. Effects of tumor necrosis factor-alpha on human sperm motility and apoptosis. J Clin Immunol 2007;27:152_62.

28. Micu M.C., Micu R., Surd S., Gîrlovanu M., Bolboacă S.D., Ostensen M. TNF- $\alpha$ inhibitors do not impair sperm quality in males with ankylosing spondylitis after short-term or long-term treatment. Rheumatology (Oxford) 2014;53(7):1250-5.

29. Tengstrand B., Carlström K. \& Hafström I. Bioavailable testosterone in men with rheumatoid arthritis-high frequency of hypogonadism. Rheumatology (Oxford) 2002; 41: 285-289.

30. Tengstrand B., Carlström K. \& Hafström I. Gonadal hormones in men with rheumatoid arthritis-from onset through 2 years. J. Rheumatol 2009; 36: 887-892.

31. Nukumizu L.A., Goncalves Saad C., Ostensen M. et al. Gonadal function in male patients with ankylosing spondylitis. Scand $J$ Rheumatol 2012; 41:476 81. 
32. Ozgocmen S., Kocakoc E., Kiris A., Ardicoglu A. \& Ardicoglu $\mathbf{O}$. Incidence of varicoceles in patients with ankylosing spondylitis evaluated by physical examination and color duplex sonography. Urology 2001;59:919-922.

33. Almeida B.P. et al. Testicular Sertoli cell function in ankylosing spondylitis. Clin. Rheumatol 2013; 32:1075-1079.

34. Martini A.C., Molina R.I., Tissera A.D., Ruiz R.D., Fiol de Cuneo M. Analysis of semen from patients chronically treated with low or moderate doses of aspirin-like drugs. Fertil Steril 2003; 80(1):221-2.

35. O'Moráin C., Smethurst P., Doré C.J., Levi A.J. Reversible male infertility due to sulphasalazine: studies in man and rat. Gut 1984; 25: $1078-84$.

36. Levi A.J., Fisher A.M., Hughes L., Hendry W.F. Male infertility due to sulphasalazine. Lancet 1979; 2: 276-278.
37. Shin T., Kobori Y., Suzuki K. et al. Inflammatory bowel disease in subfertile men and the effect of mesalazine on fertility. Syst Biol in Reproductive Medicine 2014; 60(6):373-376.

38. Moody G.A., Probert C., Jayanthi V., Mayberry J.F. The effects of chronic ill health and treatment with sulphasalazine on fertility amongst men and women with inflammatory bowel disease in Leicestershire. Int J Colorectal Dis 1997;12:220-224.

39. Cosentino M.J., Chey W.Y., Takihara H., Cockett A.T. The effects of sulfasalazine on human male fertility potential and seminal prostaglandins. J Urol 1984; 132: 682-686.

40. Toth A. Reversible toxic effect of salicylazosulfapyridine on semen quality. Fertil Steril 1979;31:538-40. 